\title{
A Combinatorial Transparent Surface Modeling from Polarization Images *
}

\author{
Mohamad Ivan Fanany ${ }^{1}$, Kiichi Kobayashi ${ }^{1}$, and Itsuo Kumazawa ${ }^{2}$ \\ 1 NHK Engineering Service Inc., 1-10-11 Kinuta Setagaya-ku Tokyo, Japan, \\ 2 Imaging Science and Engineering, Tokyo Institute of Technology \\ fanany@nes.or.jp
}

\begin{abstract}
This paper presents a combinatorial (decision tree induction) technique for transparent surface modeling from polarization images. This technique simultaneously uses the object's symmetry, brewster angle, and degree of polarization to select accurate reference points. The reference points contain information about surface's normals position and direction at near occluding boundary. We reconstruct rotationally symmetric objects by rotating these reference points.
\end{abstract}

\section{Introduction}

The simplest way to reconstruct a rotationally symmetric object is by rotating its silhouette [1]. If the object is transparent, however, finding its silhouette is very difficult due to lack of body reflection and inter-reflection effect. On the other hand, current techniques for transparent surface modeling are neither efficient nor effective in dealing with rotationally symmetric transparent objects. Because it relies on complicated light setting aimed to illuminate the whole surface of the object and it suffered much from undesirable inter-reflection effects.

In this study, we pursue a way to obtain accurate reference points that when they are rotated will give accurate surface. To the best of our knowledge, no proposed methods elaborating the extraction of such reference points. The induction of accurate reference points is difficult because it is sensitive to the light wave length, surface's microstructure, bias index, and noise. The key ideas of our method are summarized as follows. First, it is a decision tree induction technique that simultaneously uses object's symmetry, brewster angle, and degree of polarization (DOP) to extract accurate reference points. This technique directly solves the ambiguity problem in determining correct incident angle. Second, it is not necessary to illuminate the whole surface of the object, but only the area near the object's occluding boundary. Third, it gives approximate initial condition for faster iterative relaxation by rotating the normal positions and directions. In this paper, we investigate the effectiveness of this method in reconstructing an ideal cylindrical acrylic object and a more complicated object such as a plastic coca-cola bottle filled with water shown in Figure 1.

\footnotetext{
* This work is supported by the National Institute of Information and Communications Technology (NICT) of Japan.
} 


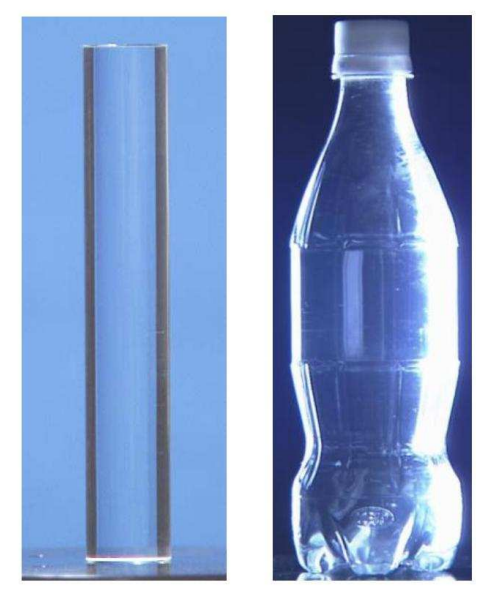

Fig. 1. Transparent objects to be reconstructed.

\section{Related works}

Transparent surface modeling is a challenging and important problem in computer vision and graphics communities. Despite recent advances in opaque surface modeling, transparent surface modeling relatively has not received much attention. Only recently, some prospective techniques for modeling transparent or specular surface based on polarization images have emerged [2-5]. These techniques, however, commonly face two fundamental difficulties. First, since transparent object has only surface reflection and little body reflection, we have to acquire as much the surface reflection as possible to infer the whole surface area. Second, since the correspondence between the degree of polarization and the obtained incident angle or surface normal is not one to one, we have to solve the ambiguity of selecting the correct value. The first difficulty, namely lack of surface reflection problem, is previously addressed by introducing very complicated light settings such as continuous spherical diffuser illuminated with many point light sources located around the sphere. Such light setting (referred to as photometric sampler firstly proposed by Nayar, et al., [6]) has two limitations: it restricts the object's diameter to be sufficiently small compared to the diffuser's diameter and it suffered much from undesirable inter-reflections. The second difficulty, namely the ambiguity problem, is previously solved by introducing other sources of information such as thermal radiation [3], or new view image [2]. The necessity of such additional information that is not readily available leads to even more impractical and time consuming implementation.

Many transparent objects around us exhibit some form of symmetry. For opaque objects, symmetry is well known of giving a powerful concept which facilitates object characterization and modeling. For instance, the implicit redundancy in symmetric models guides reconstruction process $[7,8]$, axes of symmetry provide a method for defining a coordinate system for models [9], and 
symmetries give meaningful hints for shape classification and recognition $[10$, 11]. For transparent objects, however, the significance of symmetry is largely unknown. Because the symmetry is obscured by highlights, lack of body reflection, and inter-reflections. In fact, these obscuring factors make the methods aimed for opaque surface fail to deal with transparent objects even if the objects are simpler such as those which have symmetrical properties.

Decision trees represent a simple and powerful method of induction from labeled instances [12]. One of the strength of decision tree compares to other methods of induction is that it can be used in situations where considerable uncertainty is present and the representation of the instances is in terms of symbolic or fuzzy attributes [13]. In this paper, we implement a practical decision tree induction technique based on polarization analysis, while at the same time, we avoid the difficulties faced in current transparent surface modeling techniques. Our decision tree directly resolves the ambiguity problem and produces more accurate reference vectors.

\section{Polarization analysis}

A more thorough discussion on how to obtain surface normals of transparent surface from polarization analysis of reflected light based on Fresnel equation could be found in $[5,2]$. When unpolarized light is incident on dielectric transparent surface with an oblique angle, it will be partially polarized. The total intensity of the reflected light received by camera after passing a polarizer filter is

$$
I_{s}=I_{\max }+I_{\min },
$$

where

$$
I_{\text {max }}=\frac{F_{\perp}}{F_{\perp}+F_{\|}}, \quad I_{\min }=\frac{F_{\|}}{F_{\perp}+F_{\|}} .
$$

The intensity reflectance $F_{\|}$and $F_{\perp}$ are referred to as the Fresnel reflection coefficients. They are defined as

$$
\begin{gathered}
F_{\|}=\frac{\tan ^{2}\left(\phi-\phi^{\prime}\right)}{\tan ^{2}\left(\phi+\phi^{\prime}\right)}, \\
F_{\perp}=-\frac{\sin ^{2}\left(\phi-\phi^{\prime}\right)}{\sin ^{2}\left(\phi+\phi^{\prime}\right)},
\end{gathered}
$$

where $\phi$ and $\phi^{\prime}$ are respectively incident and refraction angles. There is $\phi$ that can make $F_{\|}=0$, that is $\phi=\phi_{b}$ which is called as Brewster angle. The $\phi_{b}$ is given by $\phi+\phi^{\prime}=\pi / 2$ and Snell's law as

$$
\phi_{b}=\arctan (n),
$$

where $n$ is the bias index. 
The degree of polarization (DOP) is defined as

$$
\rho=\frac{I_{\max }-I_{\min }}{I_{\max }+I_{\min }} .
$$

For unpolarized light $I_{\max }=I_{\min }=\frac{1}{2} I_{s}$ hence $\rho=0$. When $\phi=\phi_{b}$ (Brewster angle), then $F_{\|}=0$. Hence $I_{\min }=0$ so $\rho=1$. Combining Equations (2), (3), and (5), we can rewrite the DOP as

$$
\rho=\frac{2 \sin \phi \tan \phi\left(n^{2}-\sin ^{2} \phi\right)^{1 / 2}}{n^{2}-\sin ^{2} \phi+\sin ^{2} \phi \tan ^{2} \phi} .
$$

Thus, theoretically if we know the object's bias index $n$ and $\rho$, we can estimate $\phi$, which in turn will give the surface normal $\mathcal{N}(\alpha, \zeta)$, where $\alpha$ is azimuth and $\zeta$ is zenith angles. But practically, it is difficult due to the following factors: ambiguity of estimated (see $[5,2]$ ), relation between light wave length and surface's microstructure, and noise.

\section{Light source setting}

In this study, we use five linear extended light sources (see Figure 2) putted in parallel with respect to the rotational axis of the object to be reconstructed. Such configuration is aimed to extract the boundary normal vectors. A set of reference vectors can later be chosen from these boundary normal vectors. Then we can infer the whole surface seen from the camera by rotating these reference vectors. Theoretically, we can use only one linear extended light source. But practically, there is no guarantee that using only one source would provide adequate number of boundary normal vectors due to noise and complexity of the object shape. So we suggest to use more than one light source. Considering such placement of the camera with respect to the light sources and the object, the only possible surface reflections are occurred in the left half area near occluding boundary. Contrary, the lights coming from the right half area of the surface received no polarization since most of these lights are actually transmitted instead of reflected.

Thus, if we take the simple cylindrical object and put it in this light setting, we perceive two different highlight areas, i.e., A and B, as shown in Figure 3(a). The highlights in A come from the reflection of light by near occluding boundary areas. Whereas, the highlights in $\mathrm{B}$ come from the transmission of light by the back surface. Hence, if we analyze the degree of polarization (DOP) image in Figure 3(b), we find that the DOP of area A is relatively high, but in the contrary, the DOP in B is relatively too small to be observed. Realizing such condition, we could expect that correct surface normal extractable in A. Therefore, we can rotate the surface normal obtained in A and override the surface normal in B.

The advantages of this light setting are as follows. First, it is simpler because no diffuser is needed. Second, no restriction on the diameter of the object to be reconstructed relative to the diameter of diffuser. Third, less inter-reflection is incorporated in our light setting. In omni-directional light source using diffuser, such inter-reflection shown to cause inaccurate reconstruction [2]. 

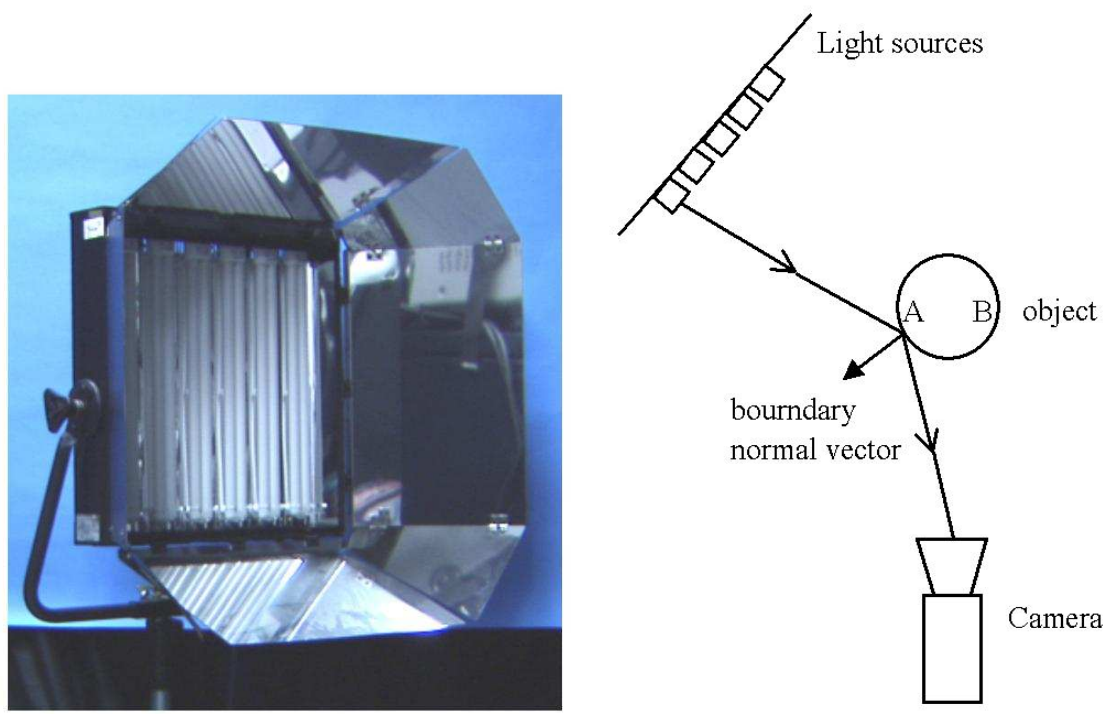

Fig. 2. Light source setting.

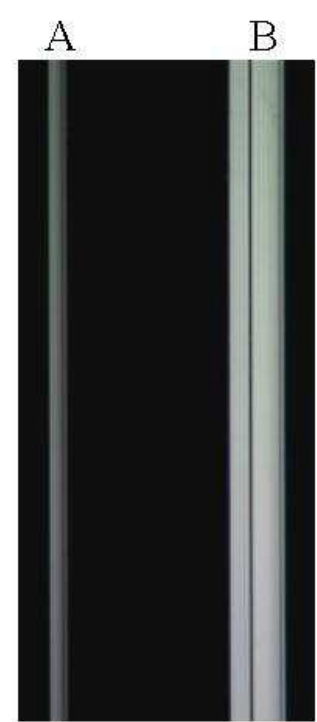

(a)

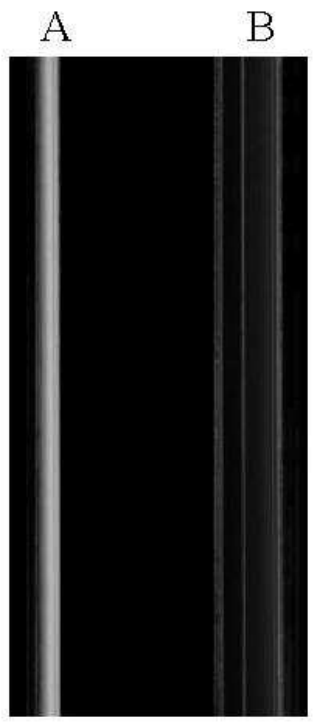

(b)

Fig. 3. The polarization image (a) and DOP image (b) of cylinder object put in our light setting. 


\section{Reference points selection}

Equation (5) allows us to measure the DOP by rotating a polarization filter placed in front of camera. By rotating the polarization filter, we obtain a sequence of images of an object. We measure from 0 to 180 degrees at 5 intervals. From this process, we obtain 37 images. We observe variance of intensity at each pixel of the 37 images. By using the least-squares minimization, we fit a sinusoidal curve to those intensities and then determine the maximum and minimum intensities, $I_{\max }$ and $I_{\min }$. For example we can observe the intensities and its fitted curves (Figure 4) of two pixels in one scanned line. We observe that smaller degree of polarization $\rho$ is more sensitive to noise, so we expect accurate surface's normal cannot be produced in these areas.
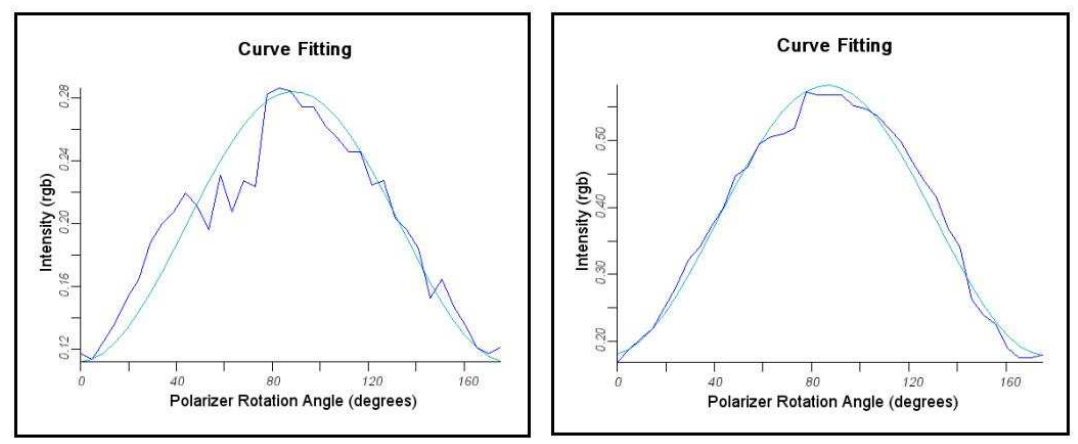

Fig. 4. Curve fitting of intensities of smaller $\rho$ (left) and higher $\rho$ (right)

Using our light source setting mentioned in Section 4 we will observe DOP image as shown in Figure 3. Since the DOP is high on specular area (the area A in Figure 3), we expect that accurate surface's normal $\mathcal{N}(\alpha, \zeta)$ in this area can be extracted. $\alpha$ is extracted by measuring the polarization rotation angle $\theta$ that give $I_{\max } . \zeta$ is extracted from Equation (6) after we know the $\rho$ (DOP) from Equation (5) and bias index $n$. In this study, we used Hi-Vision camera with long focal length and observe the zoomed in object. Thus we can assume that we observe orthographic projection image where $\zeta=\phi$ (see light reflection from the top of rotationally symmetric object in Figure 5). In addition, we also use normal density filter to reduce undesirable noise.

In a scanned line $\mathcal{L}$ in the DOP image there will be a set of points $P=$ $\left\{p_{1}, p_{2}, \cdots, p_{k}\right\}$ where the $\rho\left(p_{1}\right), \rho\left(p_{2}\right), \cdots, \rho\left(p_{k}\right) \geq \rho_{t h}$, where $\rho_{t h}$ is a given DOP threshold. We call $P$ as a set of valid points. Since the $\rho\left(p_{i}\right)$ are great we can expect to find good candidate of reference vectors there. But this does not guarantee that the extracted reference vectors or points are the ones with correct normal direction (in this case it is $\zeta$, since $\alpha$ is measured independently 


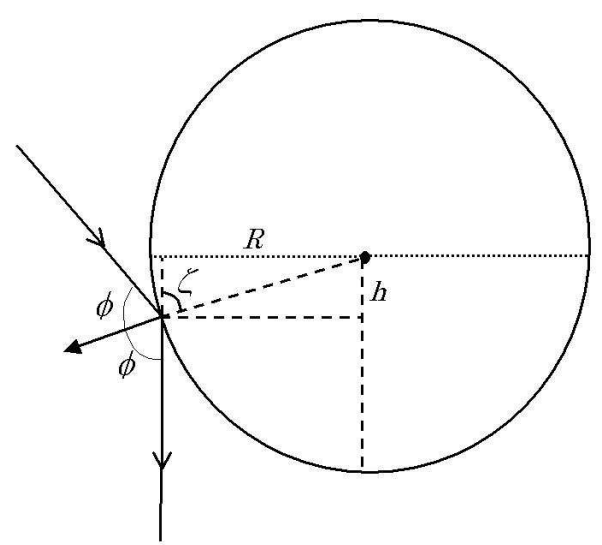

To camera

Fig. 5. Inferencing the zenith $\zeta$ and height $h$ from orthographic image

by observing $\theta$ ) and position (in this case, it is the height $h=R / \cos \zeta$ (see Figure 5).

This study pursue a way to extract such accurate reference vectors for rotation. We investigate three different ways to do this. First, the simplest way is by taking the point $p_{k}$ where $\rho\left(p_{k}\right)>$ all other points in $P$. Second, take the representational average of vector position $\bar{h}$ and direction $\bar{\zeta}$ where $\bar{h}=\Sigma h\left(p_{i}\right) / \# P$ $(i=0,1, \ldots, \# P$; where $\# P$ is the cardinality of the set $P)$ and $\bar{\zeta}=\Sigma \zeta\left(p_{i} / \# P\right)$. These two simple ways tend to generate reference vectors with wrong $h$ and $\zeta$. In the first way, the ambiguity problem is not addressed and then inconsistent $\zeta \mathrm{s}$ may be generated even though the candidate vector's pixel positions are closed. In the second way, in addition to not resolving the ambiguity problem, it averages these inconsistent candidate vectors. According to our observation the error that can be caused by the second way might be almost $0.5 R$.

After observing these two failed methods, we propose the third way, that is a decision tree induction algorithm shown in Figure 6. In this decision tree, the observed specular reflections are obtained on the half left surface area of the rotational symmetric object. When the the observed specular reflection are obtained from the half right area of the object, the second level subtree $(\zeta<$ $\phi_{b}$ and $\zeta \geq \phi_{b}$ ) should be interchanged. This decision tree directly solve the ambiguity problem by incorporating the object symmetry, brewster angle and DOP. The method is easier to understand by direct observation on how this is applied on real data sample (see Subsection 5.1).

Furthermore, the surface obtained by rotating the reference points can be used as approximate initial condition for faster relaxation to recover surface's height from gradient or needle map (Please refer to [14], pages 48-49). We can also further imposes smoothing constraint to the resulted azimuths $\alpha$ and zeniths $\zeta($ see $[15])$. 


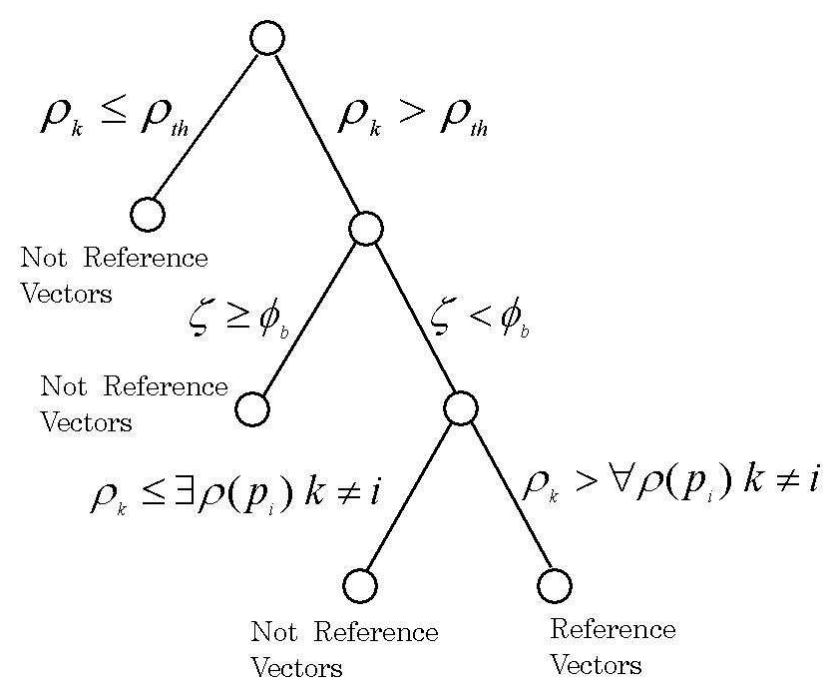

Fig. 6. Decision tree induction of referencing accurate reference points

\subsection{Direct observation}

As an example, we analyze the polarization image of cylindrical acrylic object (Fig. 3. b). The size of the image is 280 pixels width and 540 pixels height. According to a catalogue [16], the refractive index for acrylic object $1.48 \sim 1.50$. If we take the $n=1.5$ then the Brewster angle is $\phi_{b}=0.983$ radian. The DOP histogram of this image is shown in Figure 7. Let us take three arbitrary scanned lines by setting the DOP threshold to $\rho_{t h}=0.68$. We obtain two candidate vectors on a scanned line at $y=4$, four vectors at $y=10$, and three vectors at $y=140$. The azimuth and zenith angles are measured in radian. We list these candidate vectors as follows.

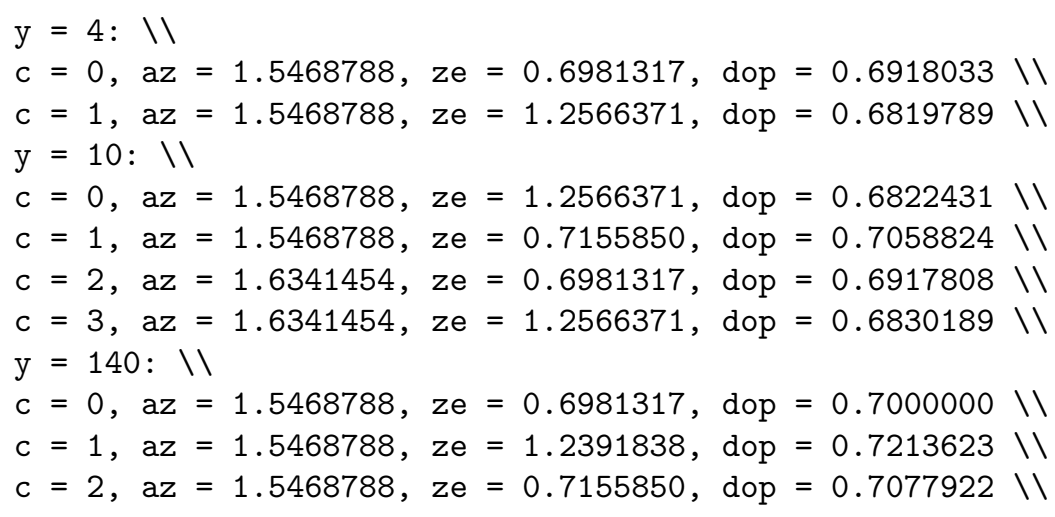

Each scanned line is processed independently. At scanned line $y=4$, we discard the candidate $c=1$ because its zenith angle is greater that Brewster angle. 
At $y=10$, we discard the candidates $c=0$ and $c=3$, and select the $c=1$. At $y=140$, we discard $c=1$, even though its DOP is the greatest among the three candidates, and we select $c=2$. At these three scanned lines, we end up with three reference vectors give two different zenith angles: $0.6981317,0.7155850$. These two angles give height estimates $h=0.77 R$ and $h=0.75 R$, which are reasonably close.

\section{DOP Histogram}

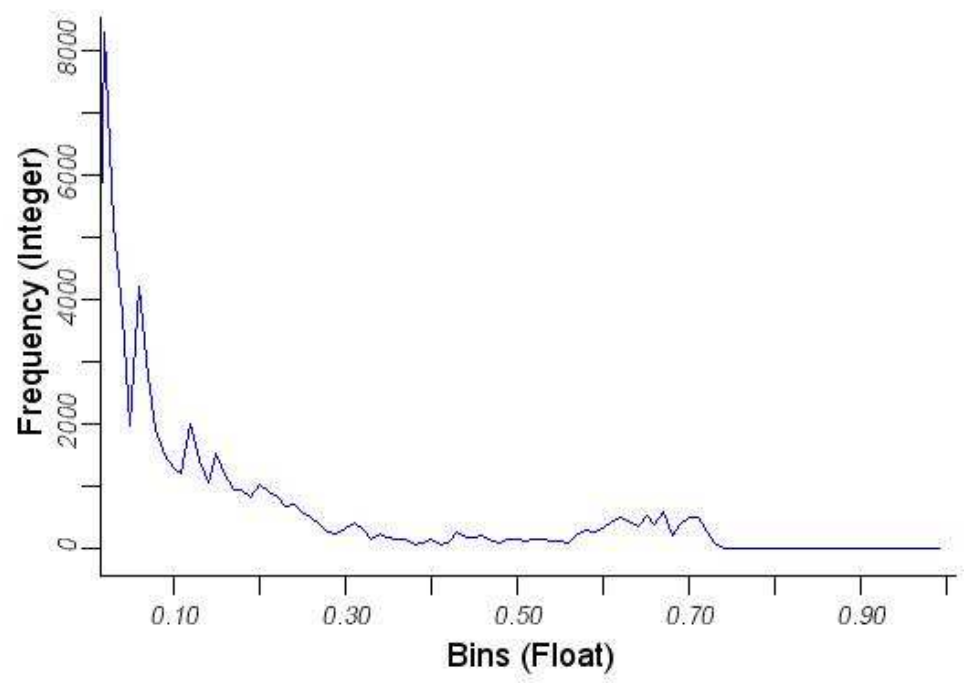

Fig. 7. The DOP histogram of Fig. 3(b)

\section{Experiments}

In this paper, we investigate the effectiveness of our method in reconstructing a simple cylindrical acrylic object and a more complicated object such as a coca-cola bottle filled with water. Beside more complex, the coca-cola bottle also contains concavities. The normal reconstruction for the two objects are shown in Figure 8. Even though we observe that the generated normals are not perfectly smooth, the estimated height from this normals shown in Figure 9 are acceptable. This reconstructed shapes are the results of relaxation procedure ([14]) by putting the shape and normals from our rotation procedure as initial conditions. By doing so, the relaxation process converges faster (in average, it needs only about 12 iterations) to a smoother and more accurate surface.

We tried to evaluate quantitatively the error generated by our methods. For the acrylic cylindrical object we measured its diameter as $3.0 \mathrm{~cm}$. For the coca- 

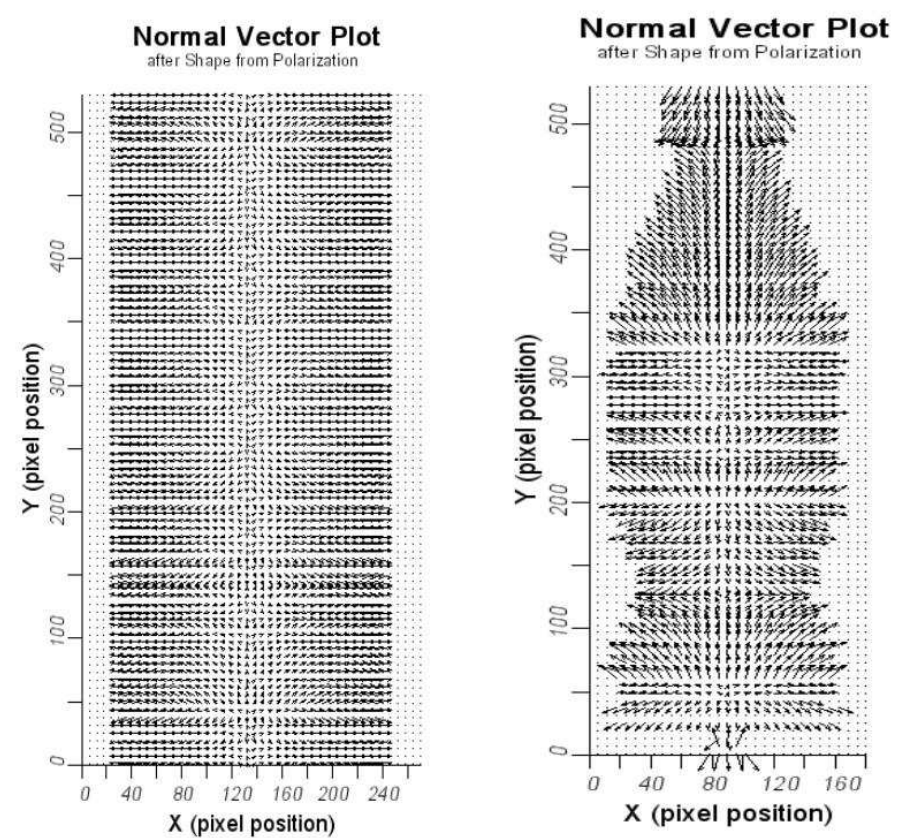

Fig. 8. reconstructed normals

cola bottle we measured several diameters of its parts. The average error for the cylindrical object is $0.038 \mathrm{~cm}$, while for the coca-cola bottle is $0.147 \mathrm{~cm}$.

\section{Conclusion}

In this paper, we present a simple decision tree induction for transparent surface modeling of rotationally symmetric objects. This decision tree allows practical induction of accurate reference vectors for rotation. The experiment results also show that our light configuration, which allows the decision tree induction is reasonably efficient and effective for reconstructing rotationally symmetric objects. The significance of this study are as follows. First, this will open ways for more practical surface reconstruction based on simple decision tree. Second, the reconstructed object could provide initial estimation that further expanded to deal with concavity and inter-reflection. Even though our method is limited to work on rotationally symmetric objects, it can also further used for non symmetric objects by extracting the symmetries contained in such non symmetric objets. In our view, doing so might be more efficient rather than directly deal with non-symmetric objects. 

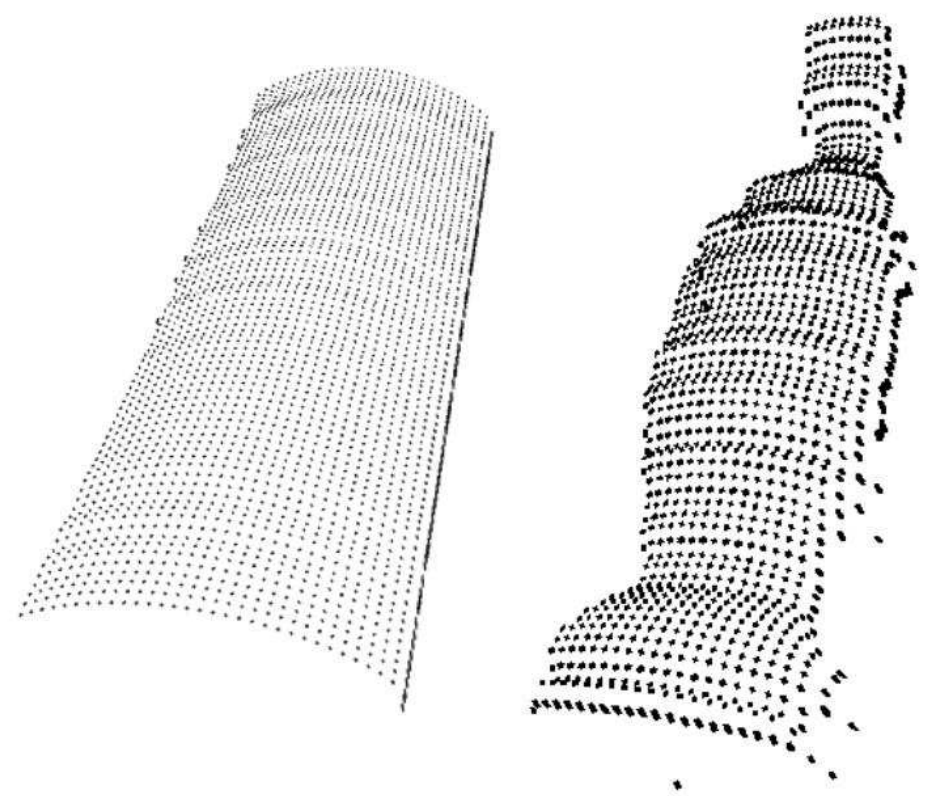

Fig. 9. Reconstructed shapes

\section{References}

1. Szeliski, R.: From images to models (and beyond): a personal retrospective. In Vision Interface '97, Kelowna, British Columbia. Canadian Image Processing and Pattern Recognition Society, (1997) 126-137.

2. Miyazaki, D., Kagesawa, M., Ikeuchi, K.: Transparent Surface Modeling from a Pair of Polarization Images. IEEE Trans. On PAMI, Vol. 26, No. 1, January (2004) 73-82.

3. Miyazaki, D., Saito, M., Sato, Y., Ikeuchi, I.: Determining Surface Orientations of Transparent Objects Based on Polarization Degrees in Visible and Infrared Wavelength. J. Opt. Soc. Am. A, Vol. 19, No. 4, April (2002) 687-694.

4. Rahmann, S., Centerakis, N.: Reconstruction of Specular Surfaces Using Polarization Imaging, Proc. IEEE Conf. Computer Vision and Pattern Recognition, (2001) $246-253$.

5. Saito, M., Sato, Y., Ikeuchi, K., Kashiwagi, H.: Measurement of surface orientation of transparent objects by use of polarization in highlight. J. Opt. Soc. Am. A/Vol. 16, No. 9 September (1999) 2286-2293.

6. Nayar, S. K., Ikeuchi, K., Kanade, T.: Determining Shape and Reflectance of Hybrid Surface by Photometric Sampling. IEEE Trans. Robotics and Automation, Vol. 6, No. 4, August, (1990) 418-431.

7. Mitsumoto, H., Tamura, S., Okazaki., K, Fukui, Y.: Reconstruction using mirror images based on a plane symmetry recovery method. IEEE Trans. on PAMI, Vol. 14, (1992) 941-946.

8. Zabrodsky, H., Peleg, S., Avnir., D.; Symmetry as a continuous feature. IEEE Trans. on PAMI, Vol. 17, (1995) 1154-1156. 
9. Liu, Y., Rothfus, W., Kanade, T.: Content-based 3d neororadiologic image retrieval: Preliminary results. IEEE International Workshop on Content-based Access of Image and Video Databases, January, (1998) 91 - 100.

10. Leou, J., Tsai, W.: Automatic rotational symmetry determination for shape analysis. Pattern Recognition 20, (1987) 571-582.

11. Wolfson, H., Reisfeld, D., Yeshurun, Y.: Robust facial feature detection using symmetry. Proc. of Int. Conf. on Pattern Recognition. (1992) 117-120.

12. Quinlan, J.R.: Induction of decision trees. Machine Learning, Vol. 1 (1986) 81-106.

13. Yuan, Y., Shaw, M.J.: Induction of fuzzy decision trees. Fuzzy Sets System, Vol. 69, (1995) 125-139.

14. Horn, B. K. P.: Height and Gradient from Shading. International Journal of Computer Vision, 5:1, (1990) 37-75.

15. Miyazaki, D., Tan, R. T., Hara, K., Ikeuchi, K.: Polarization-based Inverse Rendering from Single View. Proc. International Symposium on CREST Digital Archiving Project, Tokyo (2003) 51-65.

16. http://www.boedeker.com/acryl_p.htm 